\title{
Hydrodynamics of Droplet Impingement on a Thin Horizontal Wire
}

\author{
Jiayi Zheng $\mathbb{D}^{1},{ }^{1}$ Jing Wang, ${ }^{2}$ Yanshun Yu, ${ }^{1}$ and Taotao Chen ${ }^{1}$ \\ ${ }^{1}$ MIIT Key Laboratory of Thermal Control of Electronic Equipment, School of Energy and Power Engineering, \\ Nanjing University of Science \& Technology, Nanjing 210094, Jiangsu, China \\ ${ }^{2}$ Army Engineering University of PLA, Nanjing 210007, Jiangsu, China \\ Correspondence should be addressed to Jiayi Zheng; jyzheng@njust.edu.cn
}

Received 21 June 2018; Revised 29 August 2018; Accepted 24 September 2018; Published 18 October 2018

Academic Editor: Rama S. R. Gorla

Copyright (C) 2018 Jiayi Zheng et al. This is an open access article distributed under the Creative Commons Attribution License, which permits unrestricted use, distribution, and reproduction in any medium, provided the original work is properly cited.

\begin{abstract}
A model of droplet impacting on a thin horizontal wire is developed and numerically analyzed via level set method (LSM). Through varying the impact velocity, the flow regimes of droplet impacting on the wire are examined and analyzed. The detailed hydrodynamic behaviors including transient velocity and pressure distributions as well as the evolution of the gas-liquid interface during droplet impingement are investigated. The results indicate that the hanging mode, merging mode, and splitting mode appear during the droplet impingement on a thin horizontal wire. In merging mode, the tiny bubbles converge to big bubbles arising from the inertia difference between the liquid and the gas make. Due to the dynamic equilibrium between the friction of the thin wire and the droplet surface tension, the double reflux zone and the high-pressure zone are only observed in hanging mode rather than in merging mode and splitting mode. The flow regimes diagram depending on We and $B o$ is provided to represent the flow regimes of droplet impacting on a thin horizontal wire. The corresponding exponential correlations are given in form of $W e=a e^{b B o}$ to distinguish the boundaries between these droplet flow regimes.
\end{abstract}

\section{Introduction}

The phenomenon of droplet impingent on a solid or liquid surface exists ubiquitously in industrial processes and daily life, including rain dropping, ink-jet printing, pesticide, or fuel spraying [1-4]. Various flow patterns of a droplet impacting solid surfaces have been demonstrated by many experimental results $[2,5]$, i.e., spread, splash, break-up, and rebound. These patterns are strongly influenced by droplet size, physical properties of the droplets [6], droplet impact velocity, solid surface characteristics (surface contact angle, surface roughness) $[7,8]$, substrate geometry, etc. Revealing the hydrodynamic mechanism of drop impingement on a solid surface and prediction related flow patterns have aroused wide scientific interest in recent years for the possible manipulation.

Droplet dynamics contain many aspects, the droplet deformation $[9,10]$, the interaction between droplets and objects, bifurcation flow [11], etc. Most research has focused on the experimental observation of droplets falling on flat surfaces [12,13], and this domain has been extensively investigated in depth for decades. However, in practical applications, droplets usually impact arbitrarily shaped surfaces. Several experimental research studies and numerical simulations concerning the impact of droplets on curved surfaces [14,15], holes [16], knots [17], stairs [18], and porous media [19] as well as thin wires or fibers have also been carried out. The interactions between droplets and thin wires or fibers take on a variety of forms; for instance, droplets slide on the junction between vertical and horizontal fibers [20]; drops can impact inclined fibers and the efficiency of water drop capture ability is dramatically increased [21]; the elastic effects of fibers have been considered by experimentally studying the dynamics of a single drop's impact on a thin flexible fiber [22, 23]. Yet, droplets dripping onto thin wires become the basis for a more complicated drippage phenomenon that could be beneficial in the microfluidics field involving droplets [24].

In the present work, we focus on a numerical simulation of the dynamic behavior of water droplets as they impact a thin rigid wire. The idea arises from an interesting natural 
phenomenon that of numerous water droplets hanging on a spider web on a rainy day or in an early morning dew. Behavior of the capture of water droplets by filaments has been successfully applied for fog harvesting in arid areas, and for filtration and condensers [25]. Lorenceau et al. [17] experimentally investigated the dynamics of drops as they impacted horizontal fibers and determined the velocity threshold from the static and dynamic aspects, including the threshold below which the drop was entirely captured. Whereas in that study the threshold velocity was measured under conditions of symmetric impact, Lorenceau et al. [26] then considered the positions of the drop's impact relative to the fiber and surprisingly found that an off-center impact enhanced the ability of the fiber to capture the drop. Sher et al. [27] experimentally studied the off-center impact of water droplets on thin horizontal wires and the amount of liquid trapped on a dry, smooth horizontal wire was determined, and a nondimensional criterion was proposed. Kim and Kim [28] experimentally identified three outcomes of collision (capture, single drop fall, and splitting) when the drop impacted a thin fiber and they developed a theoretical model to predict the residual water mass that was critically dependent on the fiber thickness, rather than the impact velocity. Moreover, the role of surface wettability on the droplet impact has been examined; it is found that the droplet impact exhibits single drop falling behavior for the hydrophilic fiber and splitting behavior for the hydrophobic fiber when they have the identical impact velocity. Indeed, plenty of two-phase flow models (e.g., volume of fluid method, level set method, phase field method, etc.) can simulate a droplet impacting on a thin wire, but the emphases of the analysis are different. Khalili et al. [29] focused on acquiring threshold radius and velocity of a drop which is entirely captured by the fiber by the VOF method for tracking the free surface motion. Pasandideh-Fard et al. [5] established a three-dimensional model to examine the rather complicated free surface flow behaviors when a droplet impinges on a tube or a wire and the computer-generated images of droplet deformation after impact agreed well with photographs. Liang et al. [16] experimentally observed a drop's impact on cylindrical surfaces with different curvature ratios, in which the cylindrical surface could be a tube or wire. Meanwhile, three-dimensional simulations using the Coupled Level Set and Volume of Fluid (CLSVOF) method were also conducted for a droplet's impact on a tube. Ma et al. [14] solve the fluid-solid coupling and complex free surface problems based on the Smoothed Particle Hydrodynamics (SPH) method. In fact, a two-dimensional model using the level set method (LSM) can perfectly describe the evolution process of droplets dripping on thin wires, because the simulation results of our models not only agree very well with the experimental results shown in Figure 4, but also agree well with the analytical solutions which are shown in Figure 3.

Compared with the experimental investigations, I think the significance of the simulation lies in the fact that it can reduce the cost and improve the efficiency of the research. More importantly, the simulation can get the data which cannot be obtained by the experiment (e.g., the detailed hydrodynamic behaviors including transient velocity and pressure distributions in our article), which is helpful to elucidate the essence and mechanism of the physical process. Few numerical efforts have been carried out to explore the dynamic behaviors of a droplet impact on a thin rigid objects. Due to the fact that deformation of a droplet impact on a thin wire is an unsteady process with complicated gasliquid interface evolution as well as the difference in physical properties and external conditions between the dispersed phase and the continuous phase, the theoretical analyses of the physical mechanism about hanging mode, merging mode, and splitting mode and the droplet capture mechanism of the thin wire remain less understood. Therefore, a LSM numerical approach is introduced to predict the interaction between a droplet and a thin wire. In particular, the detailed information including the velocity and pressure field and the gas-liquid interface evolutions of the above-mentioned three types of droplet flow regimes are illustrated and discussed; the influence factors are systematically analyzed as well. In addition, more universal results or applications are preferred; that is, we hope to get a solution of a class of problems rather than a solution of a specific problem, so the key parameters need to be dimensionless and the dimensionless flow regimes diagram depending on $W e$ and $B o$ is provided to describe the flow behavior of droplet impacting on a thin horizontal wire.

\section{Droplet Impact on Thin Wires}

2.1. Mathematical Model. For the purpose of elucidating the mechanism and evolution of the droplet flow regime, a twodimensional mathematical model for the droplet impact on a thin wire was established. Compared with the $3 \mathrm{D}$ model, the 2D model omits the interaction of the viscous force inside the droplet in the $\mathrm{Z}$ direction, that is, when the droplet impacts on the thin wire in the $3 \mathrm{D}$ model, the velocity and the impact force of the droplet are greater than that in the $2 \mathrm{D}$ model. It can be seen from the comparison of our simulation results and experiment by Kim [28] that the deformation of the droplets impacting on the thin wire in the simulation is larger than that in the experiment. However, the overall deformation trend of our simulation results in the $2 \mathrm{D}$ models not only agree very well with the experimental results, but also agree well with the analytical solutions which are shown in Figure 3. In addition, the 2D model not only can reduces the computational cost, but also clearly elucidates the problem. Therefore, we use the $2 \mathrm{D}$ model to solve the problem of droplets impacting on the thin wire.

As shown in Figure 1, a water droplet (radius $R$ ) is placed at a certain height $H$ with an initial velocity $v_{0}$ ' right above a thin wire (radius $r$ ). The dripping process of the water droplet is controlled by gravity; that is, the initial impact velocity of the water droplet on the thin wire is ensured by the height and initial droplet velocity. Periodic boundary conditions are imposed on all sides of the computational domain with the aim of eliminating the boundary effect. The surface of the thin wire is set as a wetted wall boundary condition. Although the centric impacts of droplets on a wire is symmetric, the structure of our case shown in Figure 1 is not complicated, the size of which is not very large. More importantly, modeling and calculating the whole region can more comprehensively 


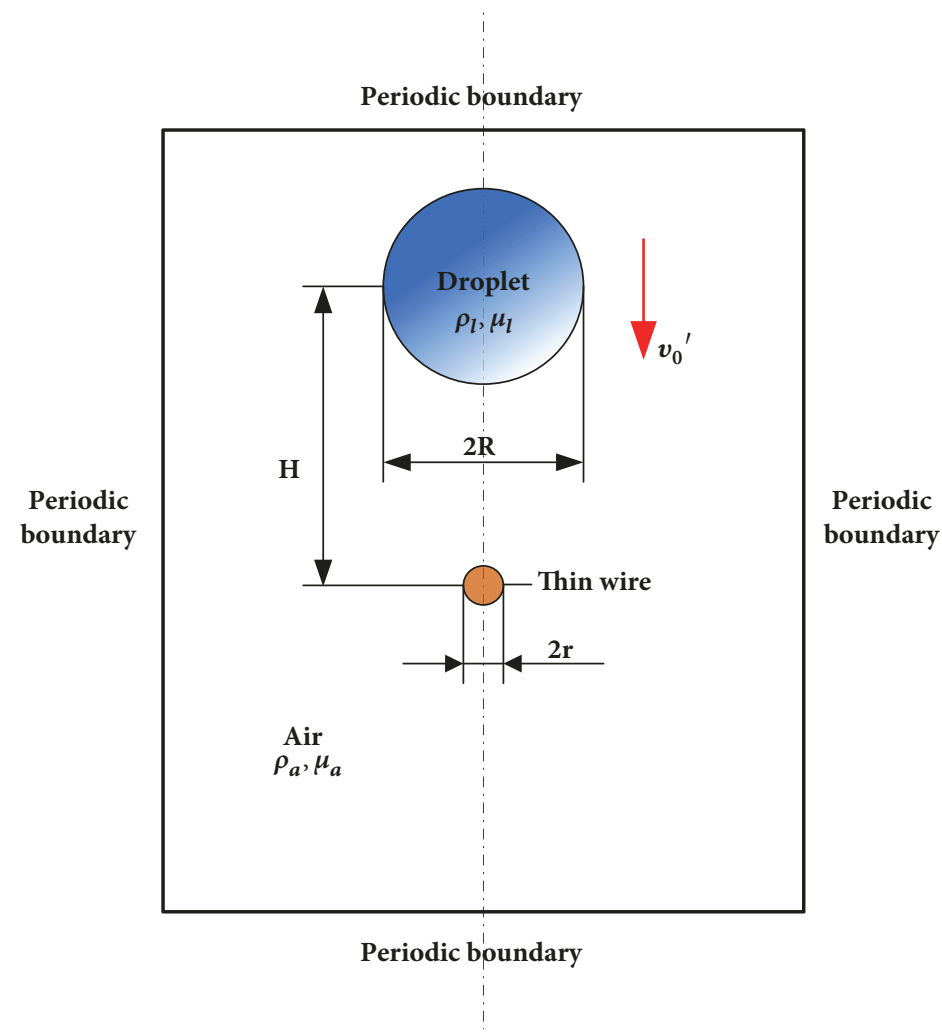

FIgURE 1: Schematic for the droplet impact on a thin wire.

capture some asymmetric details. Therefore, the whole region is calculated.

Some assumptions were made to numerically study the problem. The air and water droplets are incompressible and considered to be Newtonian fluids with constant density $\left(\rho_{\mathrm{a}}, \rho_{\mathrm{l}}\right)$ and dynamic viscosity $\left(\mu_{\mathrm{a}}, \mu_{\mathrm{l}}\right)$. The fluid flow is a laminar two-phase flow. The interfaces are tracked by the level set method, which is an Eulerian approach using a smooth function to implicitly describe the interface for the two-phase flow. The level set function $\varphi$ is defined as a signed distance to the interface. Accordingly, the interface is the zero level set in a two-phase flow system.

$$
\varphi(x)= \begin{cases}+|d| & \text { if } x \in \text { air } \\ 0 & \text { if } x \in \text { interface } \\ -|d| & \text { if } x \in \text { water }\end{cases}
$$

where $d$ is the distance from the interface. The level set method uses level set function $\varphi$ and Heaviside function $H$ to describe physical properties of fluid, i.e., $\rho$ and $\mu$ across the interface, which can be treated as follows:

$$
\begin{aligned}
& \rho=\rho_{a}+\left(\rho_{l}-\rho_{a}\right) H_{\varepsilon}(\varphi(x)), \\
& \mu=\mu_{a}+\left(\mu_{l}-\mu_{a}\right) H_{\varepsilon}(\varphi(x)),
\end{aligned}
$$

where subscript $a$ and $l$ represent the air and liquid droplet, respectively.
The Heaviside function $H$ is defined as

$$
H_{\varepsilon}(d)= \begin{cases}0 & \text { if } d<-\varepsilon \\ \frac{(d+\varepsilon)}{(2 \varepsilon)}+\frac{\sin (\pi d / \varepsilon)}{(2 \pi)} & \text { if }|d| \leq \varepsilon \\ 1 & \text { if } d>\varepsilon\end{cases}
$$

where $\varepsilon$ is a minor regularization parameter which determines the thickness of the interface. The Heaviside function can also be used as a method and index to distinguish the types of media in the calculation area.

The gas-liquid interface and liquid-solid interface were obtained by solving the convection diffusion equation of the level set function $\varphi$ and the time-dependent incompressible Navier-Stokes equations.

$$
\frac{\partial \varphi}{\partial t}+\mathbf{u} \cdot \nabla \varphi=\gamma \nabla \cdot\left[\varepsilon \nabla \varphi-\varphi(1-\varphi) \frac{\nabla \varphi}{|\nabla \varphi|}\right]
$$

where $\gamma$ means the amount of initialization and $\mathbf{u}$ is the fluid interface moving velocity vector governed by the mass and momentum equations:

$$
\begin{aligned}
\nabla \cdot \mathbf{u} & =0 \\
\rho\left(\frac{\partial \mathbf{u}}{\partial t}+\mathbf{u} \cdot \nabla \mathbf{u}\right)= & \nabla \cdot\left[-p \mathbf{I}+\mu\left(\nabla \mathbf{u}+(\nabla \mathbf{u})^{T}\right)\right]+\rho \mathbf{g} \\
& +\mathbf{F}_{s t},
\end{aligned}
$$


where $p$ is the fluid pressure, $\mathbf{I}$ is the identity matrix, $\mathbf{g}$ is the acceleration of gravity, $\mathbf{F}_{s t}$ is the surface tension, and $\mathrm{S}=$ $\rho \mathbf{g}+\mathbf{F}_{s t}$ is referred to as the source item of the equation. The surface tension is described as the following equation:

$$
\mathbf{F}_{s t}=\nabla \cdot\left\{\left[\sigma\left(\mathbf{I}-\mathbf{n} \mathbf{n}^{T}\right)\right] \delta\right\},
$$

where $\sigma$ is the surface tension coefficient, $\delta$ is the Dirac delta function which is zero everywhere except at the interface, and $\mathbf{n}$ is the unit normal of the interface that can be expressed as follows:

$$
\mathbf{n}=\frac{\nabla \varphi}{|\nabla \varphi|} .
$$

In the simulation, the ambient temperature is constant, thus physical parameters related to the temperature are constant. If not specifically indicated, the surface of the thin wire is hydrophilic and the surface contact angle is $30^{\circ}$. The paper mainly takes the droplet capture of the thin wire as the application background. The commonly used thin wire is made of copper and the contact angle of water on the copper surface is about $30^{\circ}$. For the reason, the numerical simulation of the droplet impacting on the thin wire was carried out in a typical case (contact angle is $30^{\circ}$ ). The ambient air is considered to be dynamically inactive as the droplet is falling.

2.2. Numerical Method. The finite element method is employed to solve the level set equation and Navier-Stokes equations in a two-dimensional Cartesian coordinate system. The interface convective equations of the level set method must be initialized before the transient solving. In the initialization step, the interface convective equation is solved at stationary conditions to make sure the level set parameter $\varphi$ can vary smoothly across the initial interface [30].

Triangular mesh elements (unstructured mesh) are utilized throughout the computational region. As shown in Figure 2, the grid arrangement should maintain the principle that the path of the droplet is drawn with the finer meshes, especially around the interaction interface between the water droplet and the thin wire. The mesh size of other surrounding regions gradually increases from the droplet path to the periodic boundaries to reduce the computational expense. Considering the mesh size directly affects the accuracy of the numerical calculation, so it is necessary to carry out a grid independence test with a series of mesh sizes. The sensitivity analysis of mesh size is conducted with different mesh number of Cells Per droplet Radius (CPR) (including 1,876, 5,184, $11,278,44,242$, and 176,868 ) to ensure that the numerical results are independent of the mesh size. The calculation results showed that the relative error of the average velocity of droplet at $8 \mathrm{~ms}$ is less than $1.7 \%$ when the mesh number of CPR exceeds 11,278. Therefore, considering the tradeoff between computational time and simulation precision, the mesh number of 11,278 is selected in the simulations.

Except for the mesh quality, the thickness of the interface should be thin. This is to say, the interface should be distinct enough to achieve a sharp transition, which also greatly affects the convergence of the numerical solutions; thus, it will track the fluid properties of the interface more accurately, i.e., density, dynamic viscosity, and surface tension [31].
2.3. Model Verification. The deformation of a single emulsion in shear flow which is a classic theoretical estimate based on static analysis of a viscous flow field is used to validate the present model. Taylor [32] achieved the theoretical solution (shown in (10)) of the deformation coefficient $D=(L-B) /(L$ $+B$ ) when $C a \ll 1$ ( $C a$ is the capillary number).

$$
D=\frac{19 \lambda+16}{16 \lambda+16} C a,
$$

where $\lambda$ is the viscosity ratio between the drop and the continuous phase. Figure 3 shows good agreement between the simulation results and the theoretical solutions; therefore, it fully demonstrates that the present model is rational and reliable.

According to different impact velocity and fiber diameter, three outcomes of the droplet impact on a fiber are illustrated in Figure 4; the overall deformation trend of our simulation results agree very well with the experimental results [28]. The rationality of the model is further proved.

\section{Results and Discussion}

3.1. Impact Behaviors. For a thin wire with a curvature that is relatively large, the behavior of the droplet impact upon the thin wire shows greater differences compared to impact on flat surfaces. In this work, with the increase of impact velocity, three types of droplet impact modes are obtained. As shown in Figure 5, when a droplet impacts a thin wire with a certain velocity due to gravity and the droplet is hung on the thin wire, it is called hanging mode. As the impact velocity increase, the friction and the viscous force between the droplet and the thin wire cannot overcome gravity and the inertial force of the droplet; hence the droplet falls through the thin wire and then merges into a whole which is shown in Figure 7: this is known as merging mode. Eventually, the continuously increasing impact velocity results in the inertial force overwhelming the viscous force. Then the droplet is split into two parts by the thin wire as shown in Figure 10, which is regarded as splitting mode.

The above three processes in the evolution of the morphology of the droplet can be divided into three stages (see Figures 5, 7, and 10). The first two stages, free falling and splitting, are almost identical. In the free falling stage, the droplet is a freely falling body due to gravity. Once contacting the thin wire, it begins to be split by the wire. Because the velocities of the droplet impact on the thin wire are different, the different deformation behaviors of the droplets are mainly reflected in the third stage, that is, oscillation in hanging mode, merging in merging mode, and splitting in splitting mode.

In order to analyze the characteristics of the detailed flow dynamics for the three droplet impact modes, the velocity and pressure distributions of a droplet are illustrated in Figures 6,8 , and 11, respectively. Meanwhile, three types of flow regimes diagrams on the droplet impact on a thin wire are obtained as a function of the dimensionless criterion number We and Bo shown in Figure 12. The droplet impact velocity and the surface tension of the droplet are the key parameters to control the droplet morphology. 


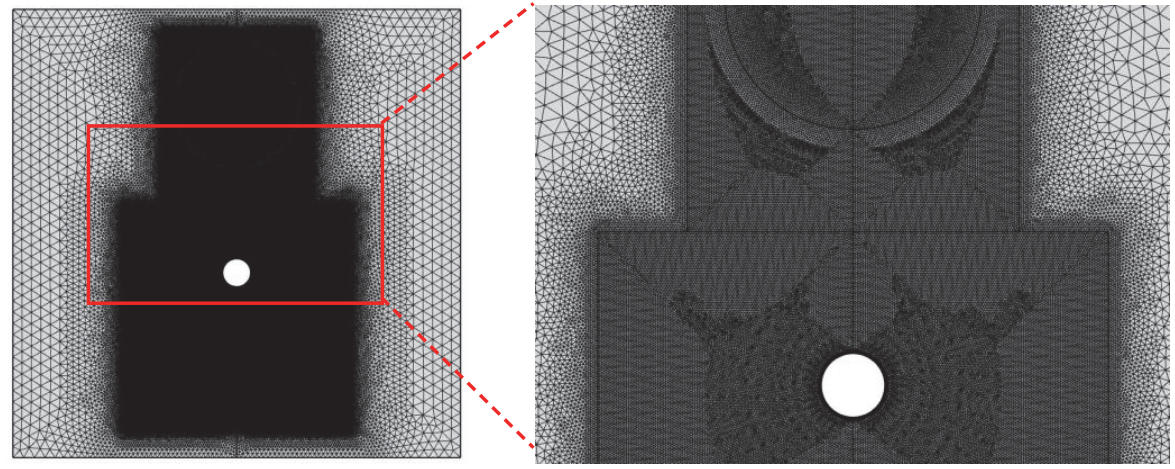

FIGURE 2: Mesh arrangement and local mesh enlargement.

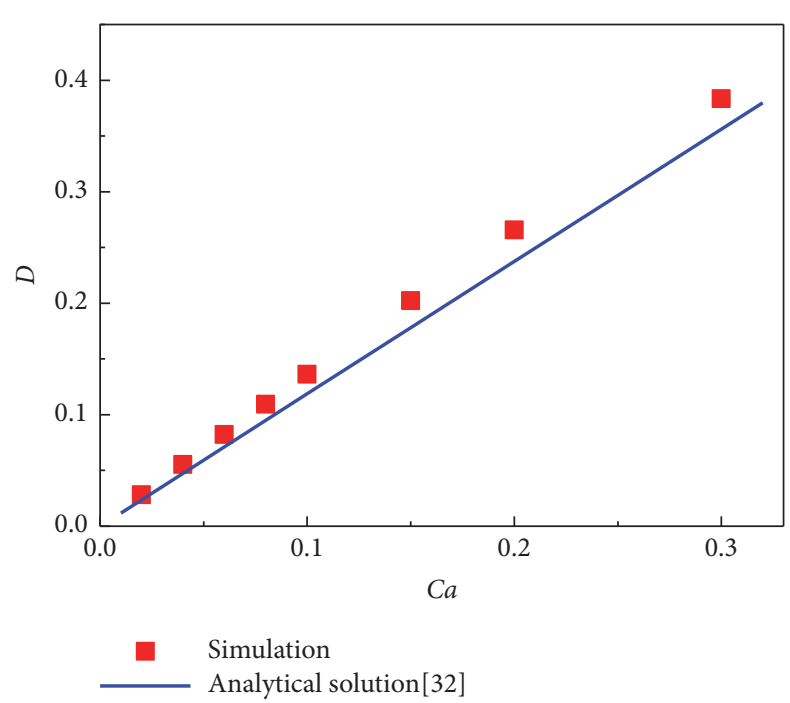

FIGURE 3: Comparison of droplet deformation in shear flow between our model and the theoretical results.

\subsection{Hydrodynamics of Droplet Impact}

3.2.1. Hanging Mode. Figure 5 shows the droplet morphology evolution in hanging mode. Before its impact upon the thin wire, the droplet is in the free fall stage. It keeps its round shape because of its low impact velocity and small droplet size. When the droplet is falling onto the thin wire, it begins to be flattened at about $6 \mathrm{~ms}$, the shape changes from a nearly round shape $(6 \mathrm{~ms})$, umbrella shape $(8 \mathrm{~ms})$, to a dumbbell shape (10 ms and $12 \mathrm{~ms}$ ). During the splitting stage, the surface tension plays a key role, which makes the droplet does not penetrate the thin wire. Next, in the oscillation stage, the inertial force continues to increase, the surface tension is unable to maintain the continuity of the interface, and the droplet passes through the thin wire at $14 \mathrm{~ms}$. At this time, the sum of the upward friction and surface tension is equivalent to the sum of the downward inertial force and gravity. Hence, the droplet is in an oscillating state; the droplet is elongated downward to the limit (20 ms) and then rebounded upward; and it moves like this repeatedly until it hangs on the thin wire statically.
In order to better understand the droplet impact mechanism, the pressure and velocity field distributions of the typical shapes are presented in Figure 6. When the droplet just impacts the thin wire (see Figure 6(a)), the falling droplet causes a local vortex flow in the surrounding air (see Figure 6(a-2)), and the high-pressure zone is near the top of the thin wire (see Figure 6(a-1)). With the increase of deformation degree, the droplet velocity is further reduced and the high-pressure zone is on both lower sides of the droplet (see Figure 6(b)), since the surface tension dominates to prevent both sides of the droplet from falling due to the inertial force and gravity. In the oscillation stage, the streamlines show a larger curvature (see Figures 6(c-2) and 6(d-2)) compared to the splitting stage, and reflux appears in the local region. In the process of elongating, the maximum pressure region appears on both sides of the droplet (see Figure 6(c-1)) to make the droplet compressed in the horizontal direction and elongated in the vertical direction, while when the droplet is elongated to the limit, the maximum pressure region appears on both sides of the thin wire (see Figure 6(d-1)), since the friction of the thin wire and the droplet surface tension have a trend towards retaining the droplet as hanging on the thin wire.

3.2.2. Merging Mode. As illustrated in Figure 7 for merging mode, compared with hanging mode, the droplet impact velocity becomes larger. The whole evolution process is very similar to hanging mode. The only difference is the third stage where the droplet falls when it passes through the thin wire, instead of hanging on the thin wire. As the droplet impact velocity increases, the inertial force of the droplet impact on the thin wire increases, and the deformation of the droplet increases accordingly. The two split parts of the droplet are elongated downward $(2 \mathrm{~ms} \sim 3.5 \mathrm{~ms})$. The gap between the two parts becomes smaller and smaller until it disappears. The two split parts finally merge into one droplet underneath the thin wire, and then continue to leave the thin wire and fall (4 ms $\sim 5 \mathrm{~ms}$ ).

The pressure and velocity distribution of the typical droplet morphologies are shown in Figure 8. It can clearly be seen that the high-pressure area appears at the top of the thin wire while the low-pressure area appears on both sides of the thin wire before the droplet leaves the thin wire 


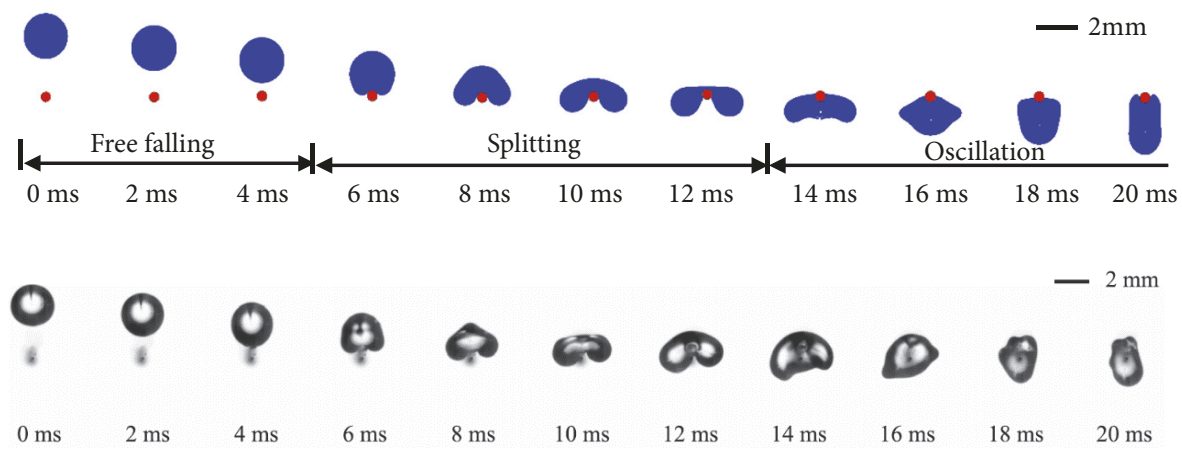

(a) Hanging mode $\left(v_{0}=0.3 \mathrm{~m} / \mathrm{s}\right.$ and $\left.r=250 \mu \mathrm{m}\right)$

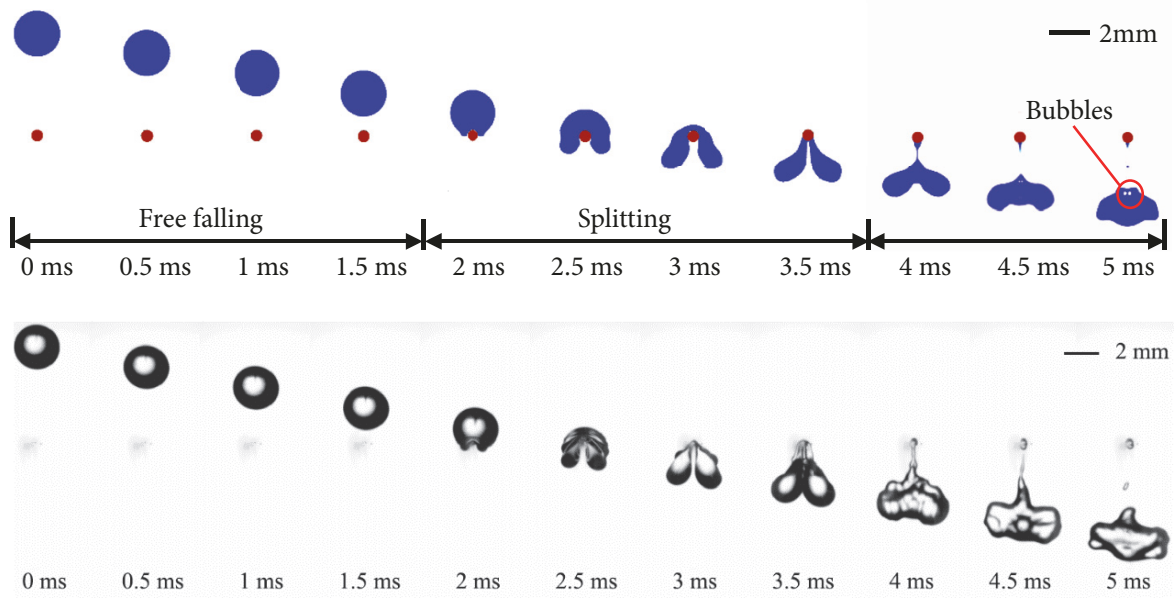

(b) Merging mode $\left(v_{0}=1.3 \mathrm{~m} / \mathrm{s}\right.$ and $\left.r=150 \mu \mathrm{m}\right)$
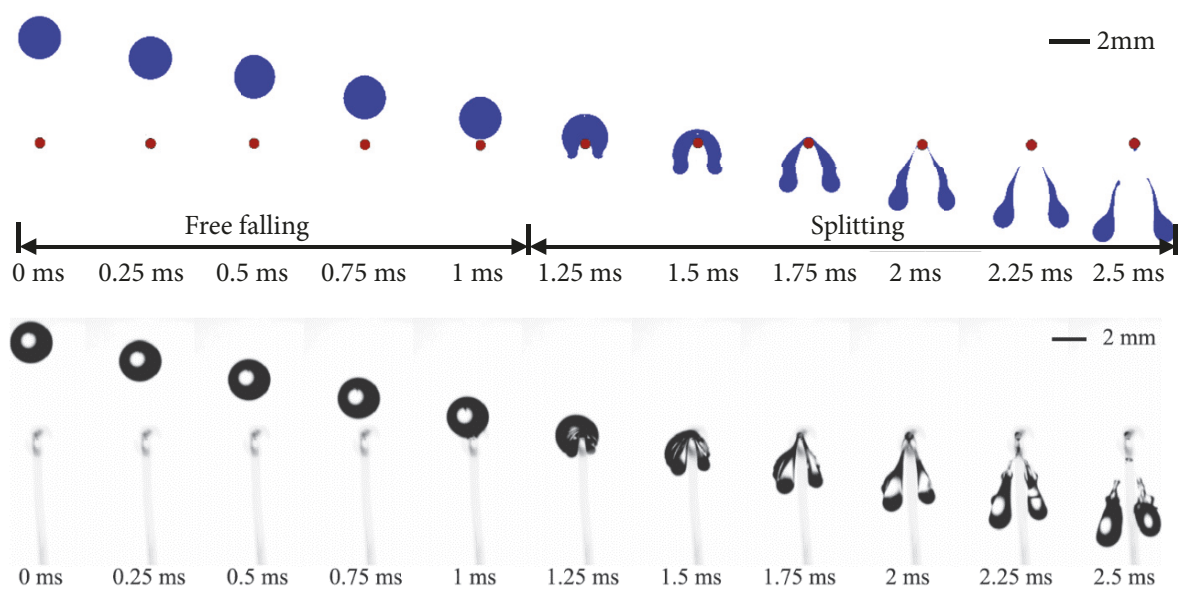

(c) Splitting mode $\left(v_{0}=2.4 \mathrm{~m} / \mathrm{s}\right.$ and $\left.r=150 \mu \mathrm{m}\right)$

Figure 4: Comparison of dynamic droplet impacting on thin wire between experiment [28] and simulation.

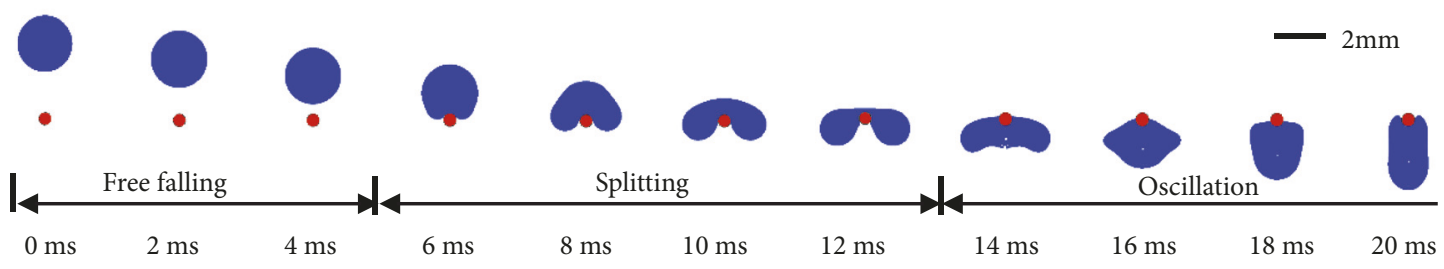

Figure 5: Time evolution for hanging mode ( $W e=1.23, B o=0.13, H=3 \mathrm{~mm}, v_{0}=0.3 \mathrm{~m} / \mathrm{s}, R=1 \mathrm{~mm}$, and $r=0.25 \mathrm{~mm}$ ). 


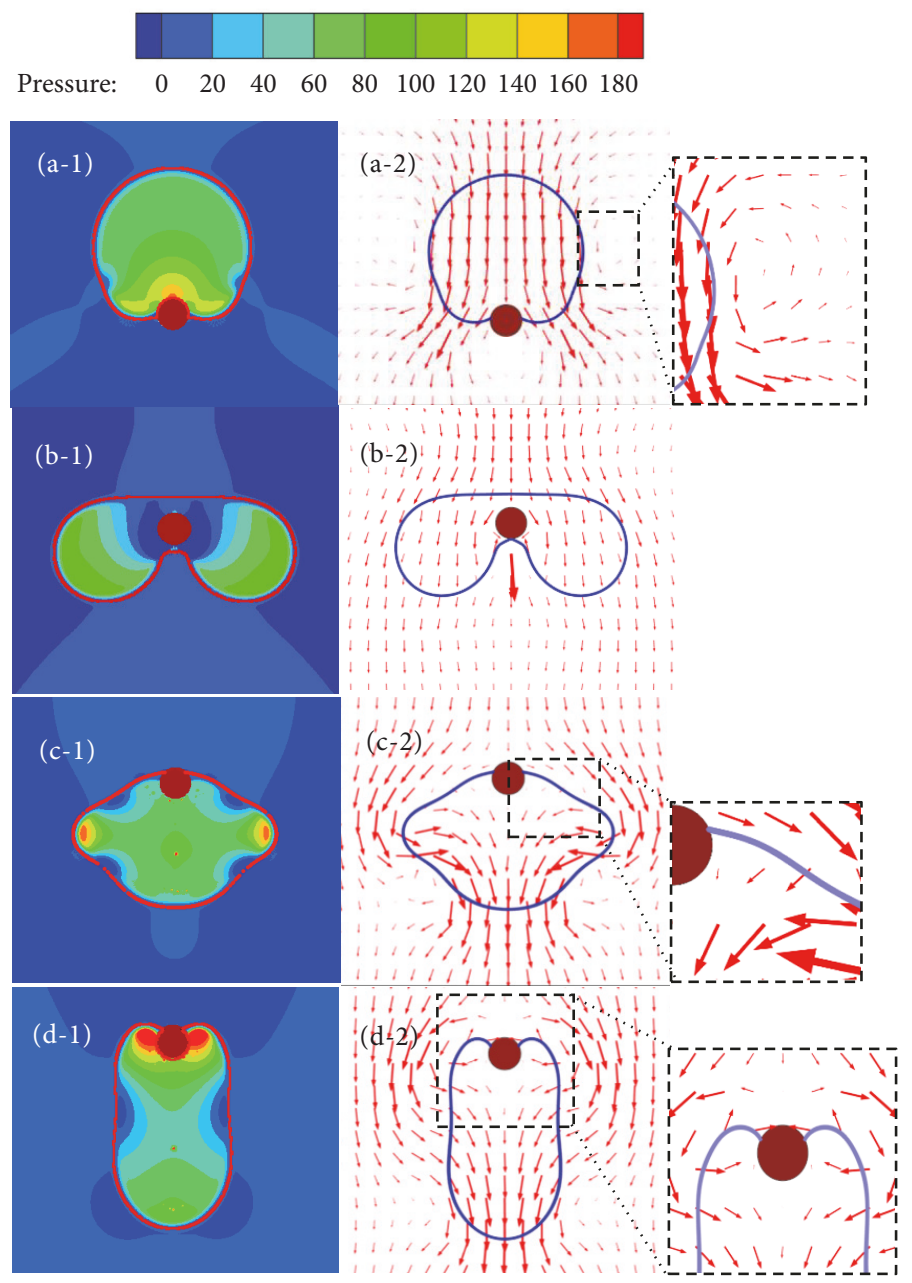

Figure 6: Pressure and velocity distribution of the droplet at (a) $6 \mathrm{~ms}$, (b) $12 \mathrm{~ms}$, (c) $16 \mathrm{~ms}$, and (d) $20 \mathrm{~ms}$.

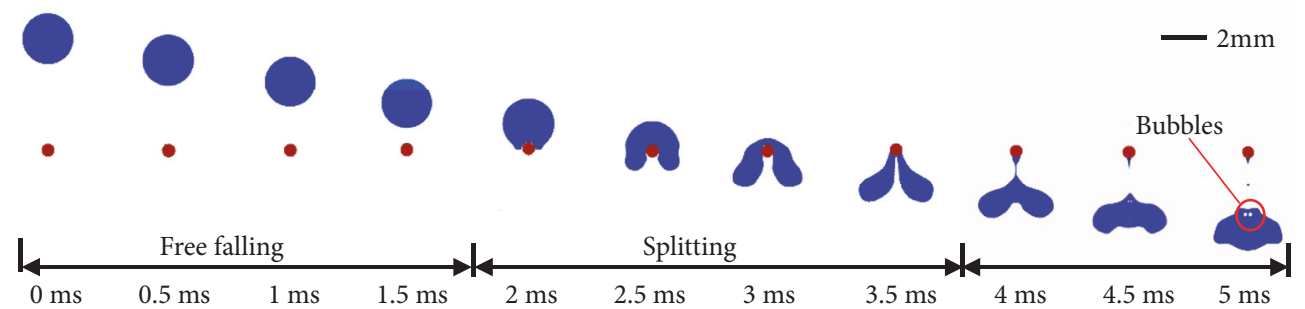

Figure 7: Time evolution for merging mode ( $W e=12.55, B o=0.04, H=4.6 \mathrm{~mm}, v_{0}=1.85 \mathrm{~m} / \mathrm{s}, R=1.1 \mathrm{~mm}$, and $r=0.25 \mathrm{~mm}$ ).

(see Figures $8(\mathrm{a}-1)$ and $8(\mathrm{~b}-1)$ ), which is mainly attributed to the friction force of the thin wire and the droplet surface tension preventing the droplet from falling due to gravity. In addition, the falling of the droplet causes a flow in the ambient air, and two vortexes appear symmetrically on both sides of the droplet (see Figures $8(\mathrm{a}-2), 8(\mathrm{~b}-2)$, and 8 (c$2)$ ). Due to the shearing action of the thin wire on the droplet, the droplet's disturbance of the surrounding airflow field is more severe; hence, the largest vortexes appear in Figure $8(b-2)$ where the shear effect is greatest. Yet, the minimum vortexes appear in Figure 8(c-2), which is because the shear effect of the thin wire disappears and the droplet just leaves the thin wire; consequently, the inertial force is relatively small, and therefore, the droplet achieves minimum velocity (Figure 8(c)). An interesting phenomenon appears in Figure 7 (e.g., $t=5 \mathrm{~ms}$ ); there are bubbles in the droplet. In fact, the formation process of the visible bubbles is that the droplet is split and then merged, during the process (e.g., $\mathrm{t}=$ $3.5 \sim 4.5 \mathrm{~ms}$ in Figure 7) a small amount of air (multiple tiny bubbles) is brought into the droplet. Because the density of air is less than that of droplet, the tiny bubbles have a tendency to move upward. At the same time, when the droplet passes through the thin wire, the flow field inside the droplet is disturbed by the friction of the thin wire, and the tiny bubbles 


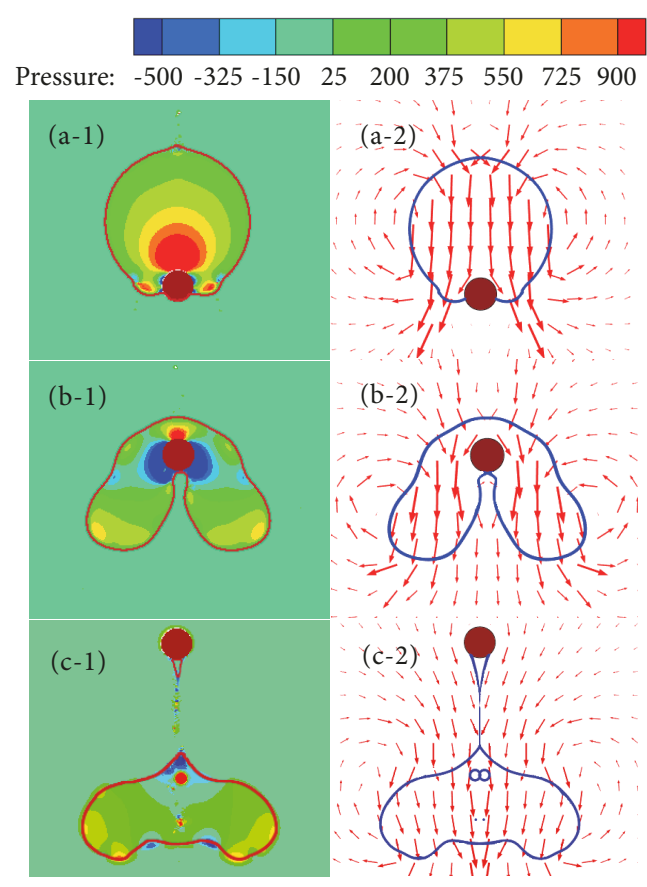

Figure 8: Pressure and velocity distribution of the droplet at (a) $2 \mathrm{~ms}$, (b) $3 \mathrm{~ms}$, and (c) $4.5 \mathrm{~ms}$.
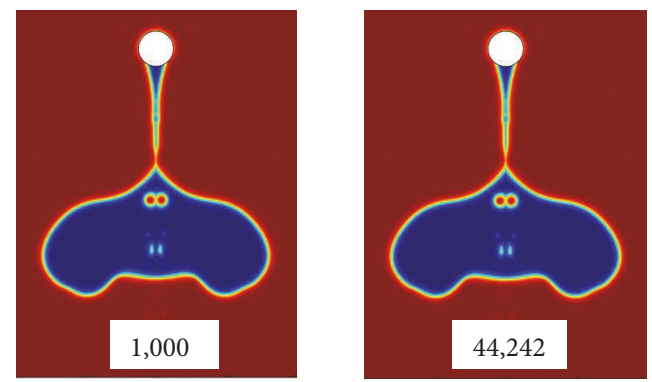

Figure 9: Bubble morphologies with different mesh sizes.

tend to move towards the middle of the droplet. Under the above two trends, tiny bubbles will merge into larger bubbles in the upper and middle position of the droplet (e.g., $t=5 \mathrm{~ms}$ in Figure 7). It appears that the process of bubble growth is actually the process of merging tiny bubbles into big bubbles. Moreover, a mesh independence test has been conducted using two different mesh number of CPR $(1,000$ and 44,242) to ensure the morphology of bubbles are independent of the mesh size. When $\mathrm{t}=4.5 \mathrm{~ms}$, there is almost no difference in the morphology of the two bubbles with different mesh sizes which are shown in Figure 9.

3.2.3. Splitting Mode. Figure 10 presents the droplet morphology evolution for splitting mode. Compared with the merging mode, the impact velocity continues to increase. The droplet is split in half $(1.25 \mathrm{~ms})$ after the free falling stage $(0 \mathrm{~ms}$ $\sim 1 \mathrm{~ms})$. The two split parts of the droplet continue to fall due to the increasing inertial force.
Three typical droplet morphologies are chosen to study the pressure and velocity distribution of the droplet (see Figure 11). With the evolution in morphology of the droplet, the size and location of the high or low-pressure area is constantly changing. The high-pressure area always appears on the top of the thin wire, and gradually decreases with the downward movement of the droplet (see Figures 11(a1)-11(c-1)). Meanwhile, for the variations in droplet shape and friction direction, the location of the low-pressure area is also constantly changing, from both sides of the thin wire (Figure 11(a-1)) to the lower (Figure 11(b-1)) and lowest position (Figure 11(c-1)). Similar to the merging mode, the maximum velocity and vortexes appear as in Figure 11(b2) while the minimum velocity and vortexes appear as in Figure 11(c-2), because the thin wire is a barrier to the droplet in Figures 11(a-2) and 11(b-2), while the thin wire has a retention effect on the droplet as in Figure 11(c-2).

3.3. Flow Regime Analysis. The droplet impact process is controlled by gravity, inertial force, surface tension, friction force, and viscous force, but the effect of viscous force can be negligible according to the present research [27]. Two nondimensional numbers are the crucial parameters to determine the droplet morphology after impact on a thin wire when the diameter ratio of the droplet and the thin wire is constant, that is, a Weber number of $W e=\rho_{l} v_{0}{ }^{2} R / \sigma$ (the ratio of inertial force to surface tension) and a Bond number of $B o=\rho_{l} g R^{2} / \sigma$ (the ratio of gravity to surface tension). Figure 12 presents a distribution diagram of the three types of flow regimes for the droplet impact on a thin wire as a function of We and Bo.

As shown in Figure 12, the hanging mode usually occurs when the Weber number (the impact velocity) is relatively small. As the short dash line shown in Figure 12, the transition from the hanging mode to the merging mode of the droplet is almost not sensitive to the Bond number when the Weber number is relatively small (less than about 9). The Bond number is inversely proportional to the surface tension of the droplet. In addition, the variation trend of the flow regime boundary between the splitting mode and the merging mode can be explained whereby for a given impact velocity, the decreasing surface tension might change the droplet behavior from splitting mode to merging mode, while for the given surface tension of a droplet, the increased impact velocity might change the droplet behavior from merging mode to splitting mode. Generally speaking, there is a greater possibility of the droplet being split by the thin wire when the impact velocity is relatively high. That is why the splitting mode is distributed in the area with the larger Weber numbers. The merging mode appears between the splitting mode and the hanging mode.

\section{Conclusions}

In this paper, a theoretical model of a droplet impact on a thin wire is developed with level set method and numerically analyzed by finite element method. The simulation produces three flow regimes (hanging mode, merging mode, and splitting mode), and their detailed hydrodynamic behaviors are systematically presented and discussed, including the velocity 


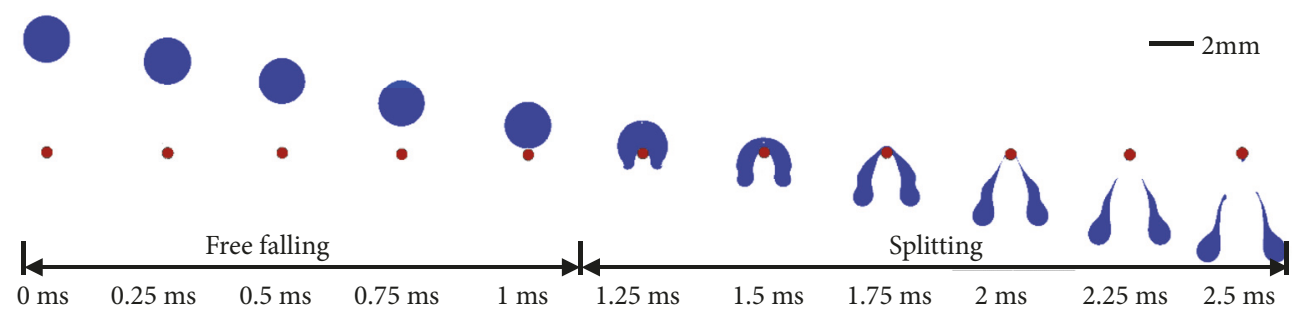

Figure 10: Time evolution for splitting mode ( $W e=44.03, B o=0.03, H=5.1 \mathrm{~mm}, v_{0}=3.9 \mathrm{~m} / \mathrm{s}, R=1.1 \mathrm{~mm}$, and $r=0.25 \mathrm{~mm}$ ).

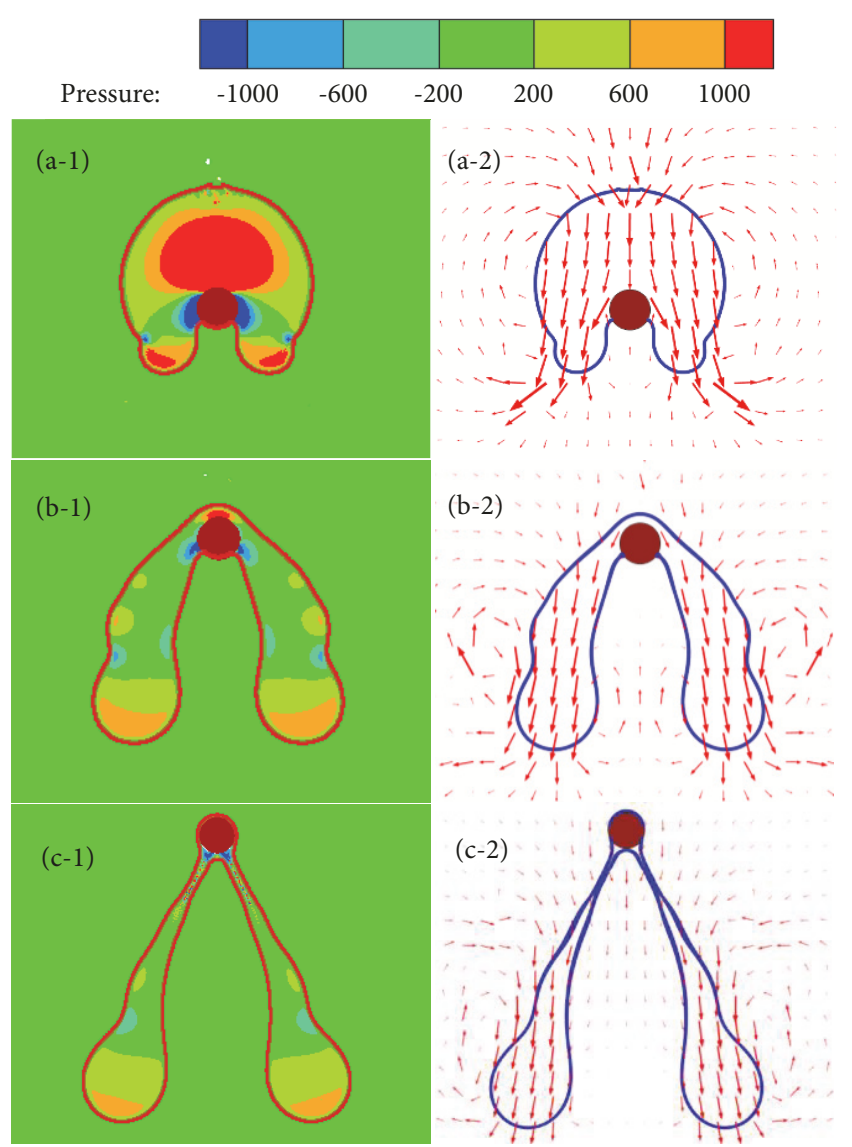

Figure 11: Pressure and velocity distribution of the droplet at (a) $1.25 \mathrm{~ms}$, (b) $1.75 \mathrm{~ms}$, and (c) $2.25 \mathrm{~ms}$.

and pressure distributions as well as the evolution of the gas-liquid interface. In merging mode, with the increase of the droplet impact velocity, an increasing number of tiny bubbles are entrained into the droplet; i.e., the tiny bubbles converge to big bubbles arising from the inertia difference between the liquid and the gas. Our results also reveal that only in hanging mode the double reflux zone and the highpressure zone are produced on both sides of the thin wire which would not happen in merging mode and splitting mode, for the friction of the thin wire and the droplet surface tension achieve an dynamic equilibrium. Meanwhile, the flow regimes diagram depending on $W e$ and $B o$ is plotted to represent the above-mentioned three droplet flow regimes. Furthermore, the corresponding exponential correlations in

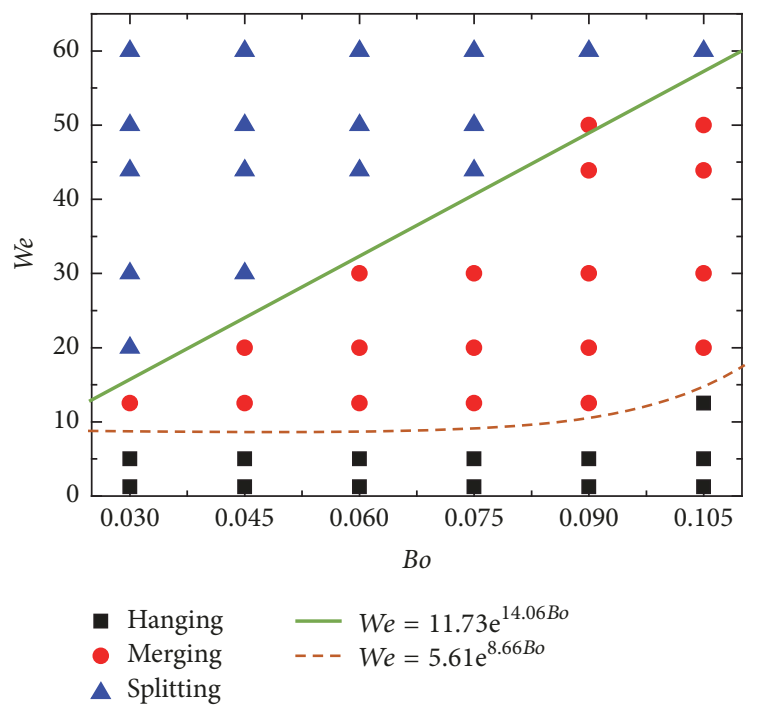

FIGURE 12: Flow regimes diagram for the droplet impact on a thin wire.

form of $W e=a e^{b B o}$ are given to distinguish the boundaries between different droplet flow regimes.

\section{Data Availability}

The data of hanging mode as an example used to support the findings of this study are included within the supplementary information files.

\section{Conflicts of Interest}

The authors declare no conflicts of interest.

\section{Acknowledgments}

This work was financially supported by the National Natural Science Foundation of China (no. 51706101), Key Laboratory of Solar Energy Science and Technology Foundation of Jiangsu Province (KLSST201704), and the Fundamental Research Funds for the Central Universities (no. 30917011328).

\section{Supplementary Materials}

This is the dynamic simulation of a droplet impacting on a thin wire from a certain distance, thus hanging on the 
thin wire which is known as hanging mode. (Supplementary Materials)

\section{References}

[1] Z.-h. Jia, W. Lei, H.-n. Yang, and G. Wang, "Dynamic Wetting Behavior of Vibrated Droplets on a Micropillared Surface," Advances in Materials Science and Engineering, vol. 2016, Article ID 8409683, 7 pages, 2016.

[2] S. Jafarmadar, B. Jalilpour, D. D. Ganji, and H. Taghavifar, "A unified model considering effects of droplet break-up and air entrainment at the initial stage of fuel spray penetration," Mathematical Problems in Engineering, vol. 2014, Article ID 127107, 11 pages, 2014.

[3] C. Zhang, Y. Chen, R. Wu, and M. Shi, "Flow boiling in constructal tree-shaped minichannel network," International Journal of Heat and Mass Transfer, vol. 54, no. 1-3, pp. 202-209, 2011.

[4] Y. Chen and Z. Deng, "Hydrodynamics of a droplet passing through a microfluidic T-junction," Journal of Fluid Mechanics, vol. 819, pp. 401-434, 2017.

[5] M. Pasandideh-Fard, M. Bussmann, S. Chandra, and J. Mostaghimi, "Simulating droplet impact on a substrate of arbitrary shape," Atomization and Sprays, vol. 11, no. 4, pp. 397-414, 2001.

[6] J. Cai, X. Hu, B. Xiao, Y. Zhou, and W. Wei, "Recent developments on fractal-based approaches to nanofluids and nanoparticle aggregation," International Journal of Heat and Mass Transfer, vol. 105, pp. 623-637, 2017.

[7] C. Zhang, Z. Deng, and Y. Chen, "Temperature jump at rough gas-solid interface in Couette flow with a rough surface described by Cantor fractal," International Journal of Heat and Mass Transfer, vol. 70, pp. 322-329, 2014.

[8] C. Zhang, Y. Chen, Z. Deng, and M. Shi, "Role of rough surface topography on gas slip flow in microchannels," Physical Review E: Statistical, Nonlinear, and Soft Matter Physics, vol. 86, no. 1, Article ID 016319, 2012.

[9] Y. Chen, X. Liu, and M. Shi, "Hydrodynamics of double emulsion droplet in shear flow," Applied Physics Letters, vol. 102, no. 5, p. 051609, 2013.

[10] Y. Chen, X. Liu, C. Zhang, and Y. Zhao, "Enhancing and suppressing effects of an inner droplet on deformation of a double emulsion droplet under shear," Lab on a Chip , vol. 15, no. 5, pp. 1255-1261, 2015.

[11] Y. Chen, C. Zhang, M. Shi, and Y. Yang, “Thermal and hydrodynamic characteristics of constructal tree-shaped minichannel heat sink," AIChE Journal, vol. 56, no. 8, pp. 2018-2029, 2010.

[12] S. Sor, A. García-Magariño, and A. Velazquez, "Rotating armbased experimental study on droplet behavior in the shoulder region of an aircraft aerodynamic surface," International Journal of Aerospace Engineering, vol. 2017, Article ID 8390905, 12 pages, 2017.

[13] V. Bertola, "An impact regime map for water drops impacting on heated surfaces," International Journal of Heat and Mass Transfer, vol. 85, pp. 430-437, 2015.

[14] Xiao-Jing Ma and Mamtimin Geni, "Simulation of Droplet Impacting on Elastic Solid with the SPH Method," Mathematical Problems in Engineering, vol. 2015, Article ID 350496, 15 pages, 2015.

[15] Z. Jin, H. Zhang, and Z. Yang, "The impact and freezing processes of a water droplet on a cold surface with different inclined angles," International Journal of Heat and Mass Transfer, vol. 103, pp. 886-893, 2016.
[16] G. Liang, Y. Guo, Y. Yang, S. Guo, and S. Shen, "Special phenomena from a single liquid drop impact on wetted cylindrical surfaces," Experimental Thermal and Fluid Science, vol. 51, pp. $18-27,2013$.

[17] É. Lorenceau, C. Clanet, and D. Quéré, “Capturing drops with a thin fiber," Journal of Colloid and Interface Science, vol. 279, no. 1, pp. 192-197, 2004.

[18] G. McHale and M. I. Newton, "Global geometry and the equilibrium shapes of liquid drops on fibers," Colloids and Surfaces A: Physicochemical and Engineering Aspects, vol. 206, no. 1-3, pp. 79-86, 2002.

[19] J. Cai, W. Wei, X. Hu, R. Liu, and J. Wang, "Fractal characterization of dynamic fracture network extension in porous media," Fractals, vol. 25, no. 02, p. 1750023, 2017.

[20] T. Gilet, D. Terwagne, and N. Vandewalle, "Droplets sliding on fibres," European Physical Journal E, vol. 31, no. 3, pp. 253-262, 2010.

[21] K. Piroird, C. Clanet, É. Lorenceau, and D. Quéré, “Drops impacting inclined fibers," Journal of Colloid and Interface Science, vol. 334, no. 1, pp. 70-74, 2009.

[22] J. Comtet, B. Keshavarz, and J. W. M. Bush, "Drop impact and capture on a thin flexible fiber," Soft Matter, vol. 12, no. 1, pp. 149-156, 2015.

[23] E. Dressaire, A. Sauret, F. Boulogne, and H. A. Stone, "Drop impact on a flexible fiber," Soft Matter, vol. 12, no. 1, pp. 200208, 2015.

[24] Y. Chen, W. Gao, C. Zhang, and Y. Zhao, "Three-dimensional splitting microfluidics," Lab on a Chip , vol. 16, no. 8, pp. 13321339, 2016.

[25] Y. Zheng, H. Bai, Z. Huang et al., "Directional water collection on wetted spider silk," Nature, vol. 463, no. 7281, pp. 640-643, 2010.

[26] E. Lorenceau, C. Clanet, D. Quéré, and M. Vignes-Adler, "Offcentre impact on a horizontal fibre," The European Physical Journal Special Topics, vol. 166, no. 1, pp. 3-6, 2009.

[27] E. Sher, L. F. Haim, and I. Sher, "Off-centered impact of water droplets on a thin horizontal wire," International Journal of Multiphase Flow, vol. 54, pp. 55-60, 2013.

[28] S. Kim and W. Kim, "Drop impact on a fiber," Physics of Fluids, vol. 28, no. 4, p. 042001, 2016.

[29] M. Khalili, H. Yahyazadeh, M. Gorji-Bandpy, and D. D. Ganji, "Application of volume of fluid method for simulation of a droplet impacting a fiber," Propulsion and Power Research, vol. 5, no. 2, pp. 123-133, 2016.

[30] H. A. Akhlaghi Amiri and A. A. Hamouda, "Evaluation of level set and phase field methods in modeling two phase flow with viscosity contrast through dual-permeability porous medium," International Journal of Multiphase Flow, vol. 52, pp. 22-34, 2013.

[31] E. Olsson, G. Kreiss, and S. Zahedi, "A conservative level set method for two phase flow II," Journal of Computational Physics, vol. 225, no. 1, pp. 785-807, 2007.

[32] G. I. Taylor, "The mechanism of plastic deformation of crystals. Part I. Theoretical," Proceedings of the Royal Society of London. Series A, vol. 145, no. 855, pp. 362-387, 1934. 


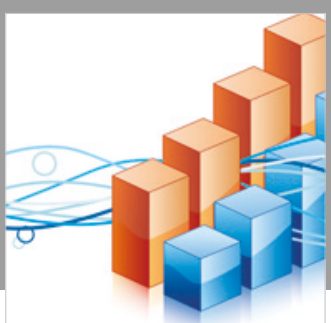

Advances in

Operations Research

\section{-n-m}
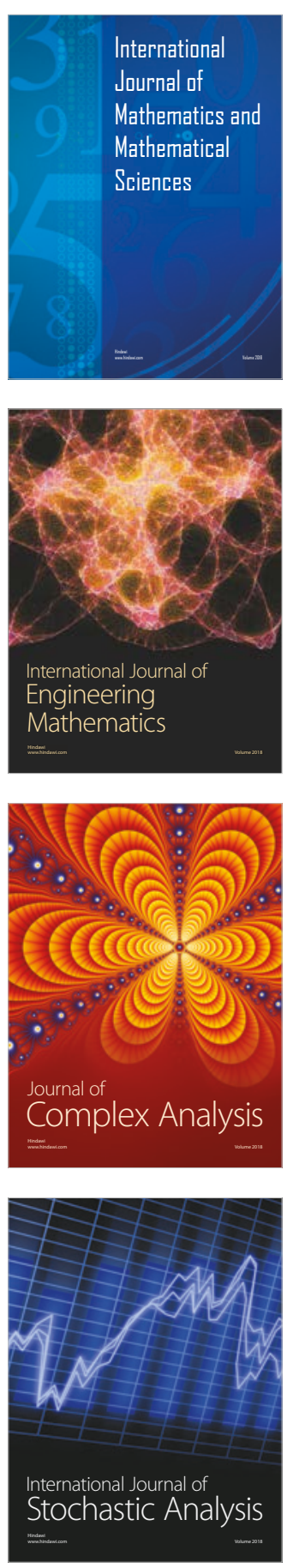
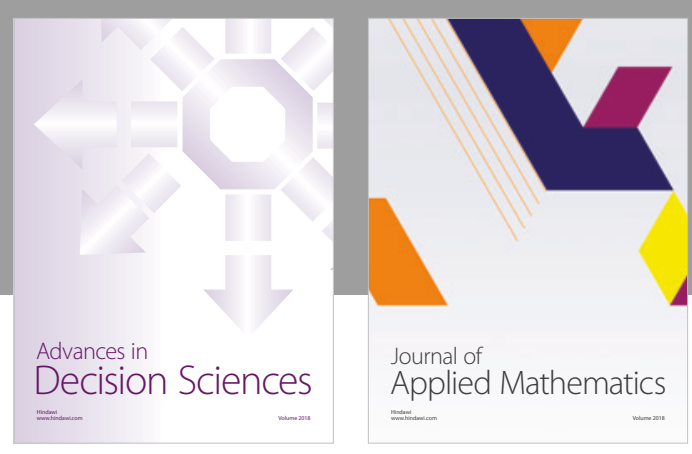

Journal of

Applied Mathematics
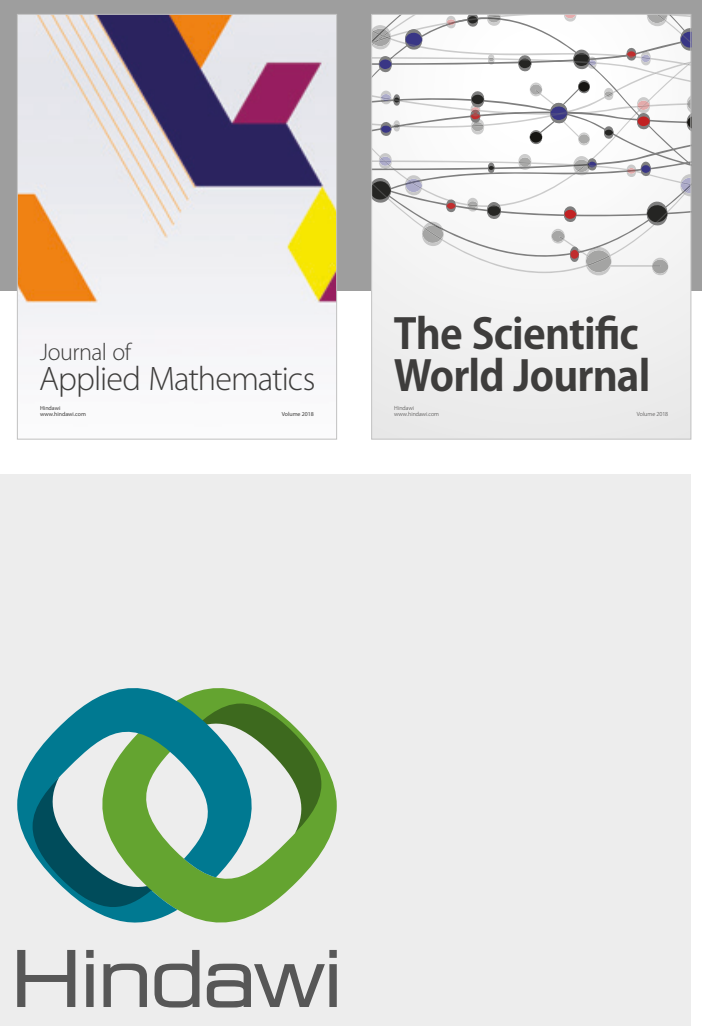

Submit your manuscripts at

www.hindawi.com

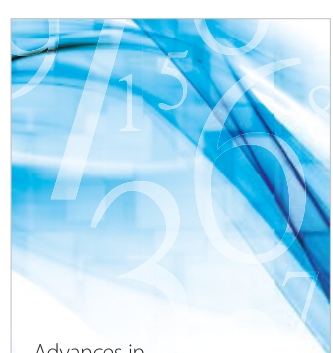

Advances in
Numerical Analysis
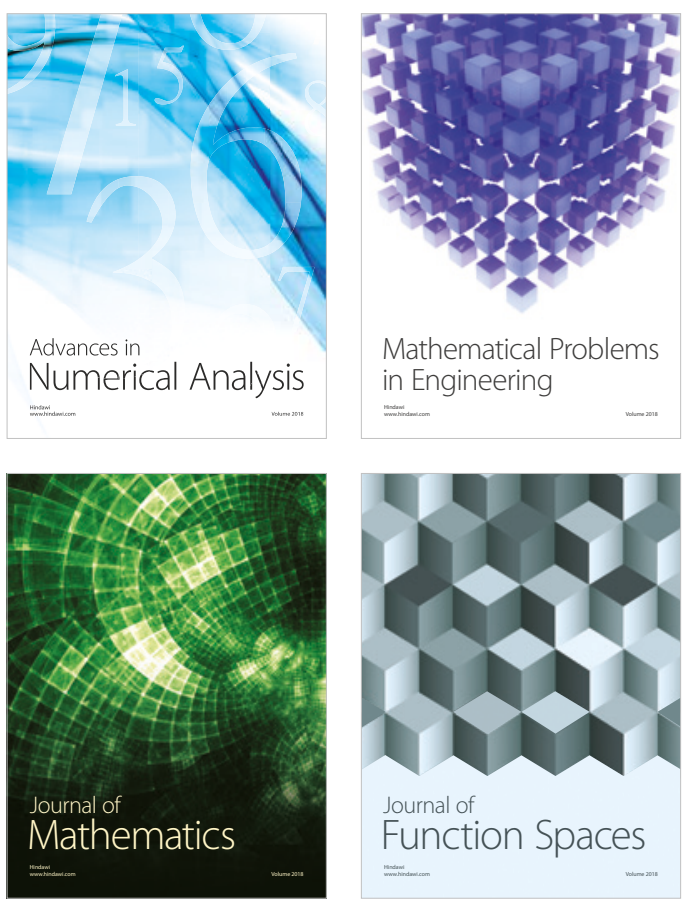

Mathematical Problems in Engineering

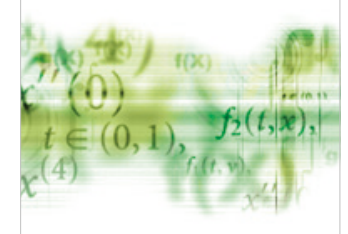

International Journal of

Differential Equations

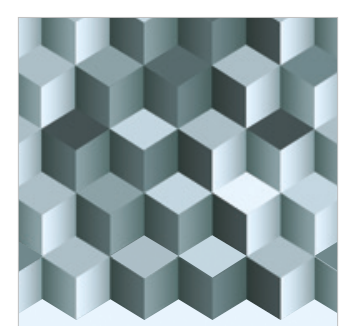

Journal of

Function Spaces
The Scientific

World Journal

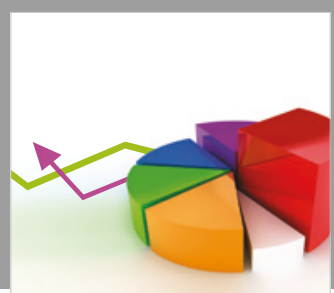

Journal of

Probability and Statistics
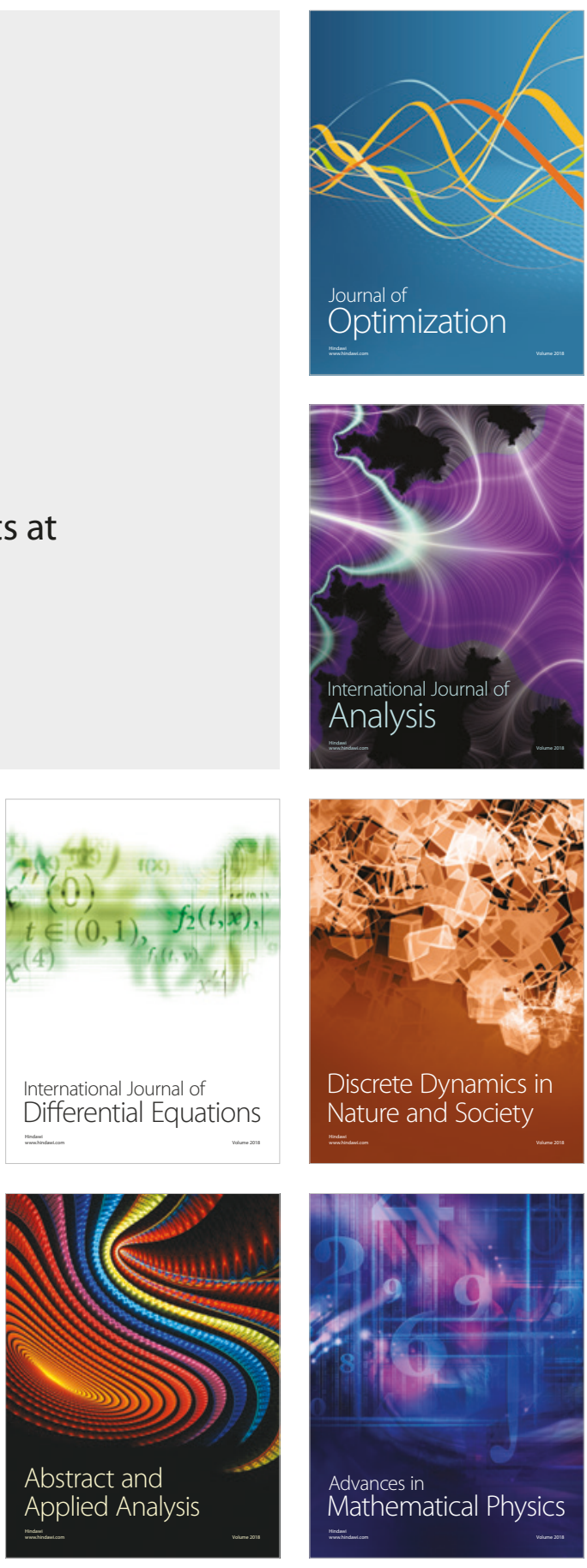